\title{
THE ORTHOCENTRIC SIMPLEX AS AN EXTREME SIMPLEX
}

\section{LEON Gerber}

Let $A$ be a variable $n$-simplex containing a fixed point $Q$ and having vertices $A_{t}$ and corresponding opposite faces $A_{1}$, $i=0,1, \cdots, n$. We use the properties of orthocentric simplexes to present brief solutions to the following problems and obtain several Erdös-Mordell type inequalities as a by-product, some of which are stronger than known inequalities.

(i) Maximize the volume of $A$ given the distances $Q A_{1}=$ $d_{i} \geqq 0, i=0, \cdots, n$.

(ii) Minimize the volume of given the distances $e_{1} \geqq 0$ from $Q$ to $\mathscr{A}_{1}, i=0, \cdots, n$.

(iii) Find the extrema of (i) and (ii) when only the power means of the distances are given.

(iv) Construct an orthocentric simplex given the lengths of the altitudes.

(v) Maximize the volume of $\mathscr{A}$ given the $(n-1)$ dimensional volumes of the faces.

(vi) Find the maximum in (i) given that $Q$ must be the centroid of $A$.

(vii) Maximize the volume of the convex hull of a skew $(n+1)$-gon given the power means of its edges.

1. Introduction. Problems (i) and (ii) have been solved recently $[1, \mathbf{1 5}]$. The present solution is much shorter and shows the relation between the two problems. Problem (iii) is a generalization of [14].

The relation between the three-dimensional version of problems (iv) and (v) was first noticed in 1773 by Lagrange [11] and the $n$-dimensional problems completely solved in 1866 by C. W. Borchardt [4]. Unaware of this latter paper, in 1953 M. A. Marmion [12] solved the three-dimensional problems anew, obtaining similar results. The present paper obtains Borchardt's results for (iv) and (v) and shows that problem (vi) and problem (vii), which generalizes the result of [13], are also related to these two.

All these solutions depend on the properties of an orthocentric simplex, that is, a simplex whose altitudes $A_{i} H_{i}, i=0, \cdots, n$, concur at its orthocenter. (In general, the altitudes of a simplex are not concurrent but are associated $[8,9]$ a weaker property.) The following facts about an orthocentric simplex $\mathscr{A}$ with orthocenter $H$ are known [7] and easily proved. Let $\mathscr{I}=\{0,1, \cdots, n\}, \mathscr{I}^{\prime}=\{1, \cdots, n\}$ and let the summa- 
tions and products $\Sigma, \Pi$ have range $\mathscr{I}$ and let $\Sigma^{\prime}, \Pi^{\prime}$ have range $\mathscr{I}^{\prime}$.

(1.1) Each edge of $\mathscr{A}$ is perpendicular to the opposite $(n-2)$-face and conversely, any simplex with this property is orthocentric.

(1.2) There exist numbers $a_{i}, i \in \mathscr{I}$, such that $A_{t} A_{j}^{2}=a_{i}+a_{1}, i \neq j$; and conversely, any simplex which has such a representation is orthocentric. Define $a^{-1}=\sum a_{1}^{-1}$. The numbers $a_{l}, i \in \mathscr{I}$, and $a$ are called the parameters of $\mathscr{A}$.

(1.3) If $H$ is interior to $\mathscr{A}$, then $a_{t}>0, i \in \mathscr{I}$, and an isometric copy of $\mathscr{A}$ can be constructed in $(n+1)$-space by locating $A_{i}$ at a distance $a_{i}^{1 / 2}$ along the $i$ th coordinate axis, $i \in \mathscr{F}$. The coordinates of $H$ are $\left(a a_{0}^{-1 / 2}, \cdots, a a_{n}^{-1 / 2}\right)$ and the distance of $H$ from the origin is $a^{1 / 2}$. If $H$ is exterior to $\mathscr{A}$, then some vertex, say $A_{0}$, is the interior orthocenter of $H A_{1} \cdots A_{n}$. In this case $a<a_{0}<0$ and $a_{1}>-a$, $i \in \mathscr{J}^{\prime}$. An isometric copy of $\mathscr{A}$ can be constructed in $(n+1)$-space by locating $H$ at a distance $(-a)^{1 / 2}$ along the zero coordinate axis and $A_{l}$ at a distance $a_{1}^{1 / 2}$ along the $i$ th coordinate axis, $i \in \mathscr{I}^{\prime}$. The coordinates of $A_{0}$ are $\left(-a_{0}(-a)^{-1 / 2},-a_{01}^{1 / 2}, \cdots,-a_{0} a_{n}^{1 / 2}\right)$ and the distance of $A_{0}$ from the origin is $\left(-a_{0}\right)^{1 / 2}$. If $H$ lies on $\mathscr{A}$, then $H$ is in fact one of the vertices, say $A_{0}$, so that the edges $A_{0} A_{i}, i \in \mathscr{I}^{\prime}$, are mutually perpendicular, i.e. $\mathscr{A}$ is a right simplex. In this case $a=a_{0}=0$ and $a_{i}>0$, $i \in \mathscr{I}^{\prime}$. An isometric copy of $\mathscr{A}$ can be constructed in $n$-space by locating $A_{0}$ at the origin and $A_{i}$ at a distance $a_{i}^{1 / 2}$ along the $i$ th coordinate axis, $i \in \mathscr{I}^{\prime}$.

Thus in particular, the parameters determine $\mathscr{A}$ to within an isometry.

(1.4) $H A_{i}^{2}=a_{1}-a, i \in \mathscr{I}$.

(1.5) $\overrightarrow{H A}_{i} \cdot \overrightarrow{H A}_{1}=a, i \neq j$.

(1.6) The volume of $\mathscr{A}$ is

$$
\begin{array}{lll}
(n !)^{-1}\left(a^{-1} \Pi a_{1}\right)^{!}, & \text {if } \quad a \neq 0, \text { and } \\
(n !)^{-1}\left(\Pi^{\prime} a_{l}\right)^{\frac{1}{2}}, & \text { if } & a=a_{0}=0 .
\end{array}
$$

(1.7) $A_{i} H \cdot H H_{i}=a$, where the segments are considered to be directed.

(1.8) A simplex is regular if and only if it is orthocentric and the $a_{t}$ are equal, $i \in \mathscr{I}$.

\section{Solution to problem (i).}

THEOREM 2.1. The maximum volume of a simplex $A$ containing the point $Q$ given the distances $Q A_{i}=d_{1} \geqq 0, i \in \mathscr{I}$, is attained by an orthocentric simplex. Assume $d_{0} \leqq d_{1} \leqq \cdots \leqq d_{n}$. If $d_{0}>0$, the parame - 
ter $a$ and the volume are determined by (2.1) and (2.2) below. If $d_{0}=0$, $\mathscr{A}$ is a right simplex and the volume is given by (2.3).

Proof. A standard compactness argument shows that the maximum is attained.

Suppose $d_{0}>0$. Let $\mathscr{A}$ be a maximizing $n$-simplex. If for some $i$, line $Q A_{i}$ is not perpendicular to $\mathscr{A}_{i}$, then the $i$ th altitude and thus the volume can be increased by moving $A_{i}$ (on a sphere of radius $d_{i}$ with center at $Q$ ) to make it perpendicular, contradicting the maximality of $\mathscr{A}$. Thus the maximum is attained when $Q$ is the orthocenter of A. By (1.4),

$$
a_{i}=a+Q A_{i}^{2}=a+d_{i}^{2}, \quad i \in \mathscr{I} .
$$

To find the parameter $a$, we need only solve the equation

$$
1=\sum a / a_{i}=\sum a /\left(a+d_{i}^{2}\right) .
$$

As $a$ increases from 0 to infinity, the right side increases monotonely from 0 to $n+1$ so there is a unique positive root. Thus the parameters are uniquely determined and the maximizing simplex is unique up to an isometry via (1.3). The maximum volume is

$$
v_{\max }=(n !)^{-1} a^{-\frac{1}{2}} \prod\left(a+d_{i}^{2}\right)^{\frac{1}{2}}
$$

and is attained when $\cos \left(A_{i} Q A_{j}\right)=a / d_{i} d_{j}$ via (1.5).

If $d_{0}=0$ and $d_{1}>0$, the simplex has $Q$ as vertex $A_{0}$ and is clearly maximized when the lines $Q A_{i}, i \in \mathscr{I}^{\prime}$, are mutually orthogonal (Hadamard inequality). The maximum is then

$$
v_{\max }=(n !)^{-1} \prod^{\prime} d_{i}
$$

(Note that the function $v_{\max }$ defined by (2.2) and (2.3) is continuous at $d_{0}=0$.) If $d_{1}=0$, the simplex is degenerate and the volume is zero.

In particular, by setting $d_{i}=R, i \in \mathscr{I}$, we have

THEOREM 2.2. The volume of an n-simplex inscribed in a sphere of radius $R$ satisfies

$$
v \leqq(n !)^{-1}(n+1)^{\frac{1}{2}(n+1)} n^{-\frac{1}{2} n} R^{n}
$$

with equality only for the regular simplex. 


\section{Solution to problem (ii).}

THEOREM 3.1. The minimum volume of the simplex. A given the distances $e_{i} \geqq 0$ from $Q$ to $\mathscr{A}_{i}, i \in \mathscr{J}$, is attained by an orthocentric simplex. Assume $e_{0} \leqq e_{1} \leqq \cdots \leqq e_{n}$. The parameter and volume are determined by (3.1) and (3.2) below.

Proof. First we prove the existence of a minimizing simplex. Let $B_{i}$ be the foot of the perpendicular from $Q$ to $\mathscr{A}_{i}$ so that $Q B_{i}=e_{i}$, and $\mathscr{A}_{i}$ is tangent at $B_{i}$ to the sphere $\mathscr{S}_{i}$ having center $Q$ and radius $e_{i}, i \in \mathscr{I}$. If $e_{0}=e_{1}=0$, the minimum volume is zero. If $e_{0}=0$ and $e_{1}>0$, then the volume exceeds one half of the volume of a sphere with radius $e_{1}$. The reciprocal of the volume is a continuous function of the $B_{i}$ and hence attains its maximum since the $B_{i}$ lie in a compact set.

Let $\mathscr{A}$ be a minimizing simplex. We shall use a device of M. M. Day [6, Lemma 4.2] to show that $B_{i}$ coincides with the centroid $G_{i}$ of $\mathscr{A}_{i}$, $i \in \mathscr{I}$. Suppose, for some $i$, that $B_{i}$ and $G_{i}$ are distinct. Let $\mathscr{L}$ be the $(n-2)$-flat of points of $\mathscr{A}_{i}$ equidistant from $B_{i}$ and $G_{i}$. Then $\mathscr{A}_{i}$ can be rotated about $\mathscr{L}$ through a small angle $\theta$ to a position $\mathscr{A}_{i}^{\prime}$ which does not intersect $\mathscr{I}_{i}$. Since the moment $m$ of $\mathscr{A}_{i}$ about $\mathscr{L}$ is not zero, for small $\theta$ the decrease in volume due to replacing $\mathscr{A}_{i}$ by $\mathscr{A}_{i}^{\prime}\left(B_{1}\right.$ is moved away from $Q$ so $G_{i}$ is moved to the interior of $\mathscr{A}$ ) is approximately $m \theta$. Translating $\mathscr{A}^{\prime}$, to touch $\mathscr{Y}_{i}$ decreases the volume further. This contradicts the hypothesis that $\mathscr{A}$ was minimizing. Thus the minimum occurs when the perpendiculars from $Q$ to the faces meet them in their centroids.

The dilatation with constant $-n$ and with center at the centroid of $\mathscr{A}$ carries the centroid of each face into the corresponding opposite vertex and hence carries the perpendiculars $Q B_{1}$ into the altitudes of $\mathscr{A}$. Since these altitudes concur at the image of $Q$, it follows that $\mathscr{A}$ is orthocentric and the orientation of the perpendiculars is the same as in the solution to (i). The minimum volume is $n^{n}$ times the values of $v_{\text {max }}$. obtained by substituting the $e_{i}$ for the $d_{i}$ in equations (2.2) and (2.3). namely,

$$
\begin{aligned}
& v_{\min }=(n !)^{-1} n^{n} a^{-\frac{1}{2}} \prod\left(a+e_{i}^{2}\right)^{\frac{1}{2}}, \quad \text { if } \quad e_{0}>0 . \\
& =(n !)^{-1} n^{n} \prod^{\prime} e_{i}, \quad \text { if } e_{0}=0 \text {. }
\end{aligned}
$$

where $a$ is the unique positive root of

$$
1=\sum a /\left(a+e_{i}^{2}\right) .
$$

In particular, by setting $e_{i}=r, i \in \mathcal{I}$, we have 
THEOREM 3.2. The volume of an n-simplex with inscribed sphere of radius $r$ satisfies

$$
v=\geqq(n !)^{-1}(n+1)^{\frac{1}{2}(n+1)} n^{\frac{1}{2} n} r^{n}
$$

with equality only for the regular simplex.

Since we do not solve (2.1) or (3.2) explicitly, we obtain some bounds for the positive root of (3.2) which will prove useful later.

THEOREM 3.3. The unique positive root of (3.2) satisfies

$$
\frac{1}{n} e_{0} e_{1} \leqq a \leqq \frac{1}{k}\left(\prod_{i=0}^{k} e_{i}^{2}\right)^{1 /(k+1)}, \quad k \in \mathscr{I}^{\prime}
$$

Proof. Let $f(a)$ denote the right side of (3.2); we have

$$
f\left(\frac{1}{n} e_{0} e_{1}\right) \leqq \frac{\frac{1}{n} e_{0} e_{1}}{\frac{1}{n} e_{0} e_{1}+e_{0}^{2}}+\frac{n\left(\frac{1}{n} e_{0} e_{1}\right)}{\frac{1}{n} e_{0} e_{1}+e_{1}^{2}} \leqq 1
$$

and

$$
f\left(e_{0} e_{1}\right) \geqq \frac{e_{0} e_{1}}{e_{0} e_{1}+e_{0}^{2}}+\frac{e_{0} e_{1}}{e_{0} e_{1}+e_{1}^{2}}=1 .
$$

Thus $\frac{1}{n} e_{0} e_{1} \leqq a \leqq e_{0} e_{1}$. For $k=2, \cdots, n$, let $t_{i}=e_{i}^{-2 /(k+1)}, i=0,1, \cdots, k$ and $t=\prod_{i=0}^{k} t_{i}$. Then by omitting some positive terms and using the inequality of the arithmetic and geometric means with weights $t_{t}^{k-1}$ we obtain

$$
\begin{aligned}
f\left(\frac{1}{k}\left(\prod_{i=0}^{k} e_{i}^{2}\right)^{1 /(k+1)}\right) & \geqq \sum_{i=0}^{k}(1 / k t) /\left[(1 / k t)+t_{i}^{-(k+1)}\right] \\
& =\sum_{i=0}^{k} t_{i}^{k-1} /\left(t_{l}^{k-1}+k t t_{\imath}^{-2}\right) \\
& \geqq\left[\sum_{i=0}^{k} t_{i}^{k-1}\right]^{2} /\left[\sum_{i=0}^{k} t_{l}^{k-1}\left(t_{i}^{k-1}+k t t_{i}^{-2}\right)\right] \\
& =\left[\sum_{i=0}^{k} t_{i}^{k-1}\right]^{2} /\left[\sum_{i=0}^{k} t_{l}^{2 k-2}+k t t_{i}^{k-3}\right] \\
& \geqq 1 .
\end{aligned}
$$


The last inequality follows from

$$
\begin{aligned}
{\left[\sum_{i=0}^{k} t_{i}^{k-1}\right]^{2} } & -\left[\sum_{i=0}^{k} t_{i}^{2 k-2}+k t t_{i}^{k-3}\right] \\
& =2 \sum_{o \leqq i<j \leqq h} t_{i}^{k-1} t_{j}^{k-1}-k \sum_{i=0}^{k}\left(\prod_{i=0}^{k} t_{j}\right) t_{i}^{k-3}
\end{aligned}
$$

which is positive since the exponents $(k-1, k-1,0, \cdots, 0)$ in the first term on the right majorize the exponents $(k-2,1, \cdots, 1)$ of the second term in the sense of Muirhead [10, p. 45].

We also note that Newton iteration applied to $f(a)-1$, with an initial value of zero, seems to converge rapidly.

4. Solution to problem (iii). First we maximize the volume of $\mathscr{A}$ given that the distances $d_{i}=Q A_{i}, i \in \mathscr{I}$, satisfy

$$
\frac{1}{n+1} \sum d_{i}^{p}=s^{p}
$$

for some fixed $s$ and $p>0$.

We begin by treating two special cases. If some distance, say $d_{0}$, equals zero, the maximum of (2.3) is clearly attained when $d_{i}=$ $[(n+1) / n]^{1 / p} s, i \in I^{\prime}$, so $\mathscr{A}$ is an isosceles right simplex, and the volume is given by

$$
v=(n !)^{-1}[(n+1) / n]^{n / p} S^{n} .
$$

In the second case, if $d_{i}=s, i \in \mathscr{I}$, so that $\mathscr{A}$ is a regular simplex, then $a+s^{2}=(n+1) a$ from (2.1), so $a=s^{2} / n$ and the volume is found from (2.2) to be

$$
v=(n !)^{-1} a^{-\frac{1}{2}}[(n+1) a]^{\frac{1}{2}(n+1)}=(n !)^{-1} n^{-\frac{1}{2} n}(n+1)^{\frac{1}{2}(n+1)} s^{n} .
$$

Observe that the value in (4.3) exceeds that in (4.2) provided

$$
p \geqq p_{n}=2 /\left[1+\frac{\log (n+1)}{n \log (n+1)-n \log n}\right] .
$$

(We have $p_{1}=1$ and $p_{n}<2 /[1+\log (n+1)]<1$ for $n \geqq 2$ since $\left.[(n+1) / n]^{n}<e.\right)$. We may now state

THEOREM 4.1. The maximum volume of $\mathscr{A}$ subject to (4.1) is attained only by a regular simplex and is given by (4.3) if $p$ satisfies 
(4.4). Otherwise it is attained only by an isosceles right simplex and is given by (4.2).

Proof. Again it is clear that the maximum is attained. Now the set of points $\left(d_{0}, \cdots, d_{n}\right)$ under consideration consists of that part of the surface in $(n+1)$-dimensional space defined by (4.1) and bounded by the cooordinate primes $d_{i}=0, i \in \mathscr{I}$. On the interior of this set, i.e. when no $d_{i}=0$, the maximum volume, $v_{\max }$, of $a$ simplex with these $d_{i}$ is given by (2.2). An easy application of the method of Lagrange to log $\left(v_{\max }\right)$ with (2.1) and (4.1) as constraints shows that the only relative extremum is when $d_{i}=s, i \in \mathscr{I}$. Thus if $p \geqq p_{n}$ the relative extremum (4.3) is the maximum and if $p<p_{n}$ the boundary value (4.2) is the maximum.

If (4.1) is modified so $p \leqq 0$, the volume is unbounded. In fact, for $d_{i}=d, \quad i \in \mathscr{I}^{\prime}$, arbitrarily large, we can pick $d_{0}>0$ to satisfy (4.1). Select $A_{i}, i \in \mathscr{I}^{\prime}$, so that $Q A_{1} \cdots A_{n}$ is an isosceles right simplex of volume $(n !)^{-1} d^{n}$. We can then choose $A_{o}$ so that $\mathscr{A}$ contains this simplex.

To solve the second part of problem (iii) we minimize the volume of $\mathscr{A}$ given that the distances $e_{i}$ from $Q$ to $A_{i}, i \in \mathscr{I}$, satisfy for some fixed $t>0$,

$$
\begin{gathered}
\frac{1}{n+1} \sum e_{i}^{p}=t^{p}, \text { for some fixed } p<0, \text { or } \\
\prod e_{i}=t^{n+1}
\end{gathered}
$$

(For positive $p$ we can attain a minimum of zero by setting $e_{0}=e_{1}=$ 0.) It follows from (4.5) that $e_{0}>(n+1)^{1 / p} t$, and as $e_{0}$ approaches this value, the others approach infinity as does the corresponding $v_{\min }$.

Since $e_{0}>0$, it follows from (3.4) and (3.2) that

$$
a^{n} \leqq e_{0}^{n} e_{1}^{n} \leqq e_{0}^{n} \prod^{\prime} e_{i}=e_{0}^{n-1} t^{n+1}
$$

Then

$$
n ! n^{-n} v_{\min }=a^{-\frac{1}{2}} \prod\left(a+e_{i}^{2}\right)^{\frac{1}{2}}>\left(e_{0}^{n-1} t^{n+1}\right)^{-1 / 2 n} t^{n+1}
$$

so here too the minimum is not attained for small values of $e_{0}$. Thus, via Lagrange we have

THEOREM 4.2. The minimum volume of $\mathscr{A}$ subject to (4.5) or (4.6) is attained when $e_{i}=t, i \in \mathscr{I}$, and is given by 


$$
v=(n !)^{-1} n^{\frac{1}{2} n}(n+1)^{\frac{1}{2}(n+1)} t^{n} .
$$

This completes the solution of problem (iii). Using (4.3) and (4.2) with (4.1), and (4.7) with (4.5) and (4.6) to give bounds on the volume in terms of the power means we obtain

THEOREM 4.3. In an arbitrary $n$-simplex of volume $v$, let $Q$ be a point whose distances from the vertices and faces are $d_{i}$ and $e_{i}$ respectively, $i \in \mathscr{I}$. Then

(4.8) $\left(\frac{1}{n+1} \sum d_{i}^{p}\right)^{1 / p} \geqq(n !)^{1 / n} n^{\frac{1}{2}}(n+1)^{-(n+1) / 2 n} v^{1 / n} \quad$ for $\quad p \geqq p_{n}$,

(4.9) $\left(\frac{1}{n+1} \sum d_{i}^{p}\right)^{1 / p} \geqq(n !)^{1 / n}[n /(n+1)]^{1 / p} v^{1 / n} \quad$ for $\quad p_{n} \geqq p>0$,

$$
\begin{aligned}
& \left(\frac{1}{n+1} \sum e_{1}^{p}\right)^{1 / p} \leqq(n !)^{1 / n} n^{-1}(n+1)^{-(n+1) / 2 n} v^{1 / n} \text { for } p<0, \\
& \left(\prod e_{i}\right)^{1 /(n+1)} \leqq(n !)^{1 / n} n^{-\frac{1}{2}}(n+1)^{-(n+1) / 2 n} v^{1 / n}
\end{aligned}
$$

Equality holds in (4.8), (4.10) and (4.11) only for the centroid of a regular simplex and in (4.9) only for the vertex of an isosceles right simplex.

The result of [14] is obtained by setting $p=1$ in (4.8). Combining (4.8) with (2.4) and (4.11) with (3.3) we get the following theorem, the first part of which improves the result of [3].

THEOREM 4.4. For the interior point $Q$ of a simplex of inradius $r$ and circumradius $R$,

$$
\left(\frac{1}{n+1} \sum d_{1}^{p}\right)^{1 / p} \geqq n r \quad \text { for } \quad p \geqq p_{n},
$$

and

$$
\left(\prod e_{t}\right)^{1 / n+1} \leqq R / n
$$

with equality only for the centroid of a regular simplex.

5. On the existence of simplexes with specified faces or altitudes. We shall need the following extensions of the triangle inequality to $n$ dimensions; $v_{k}$ denotes the $k$-dimensional volume function.

THEOREM 5.1. Let $f_{0} \geqq f_{1} \geqq \cdots \geqq f_{n}>0$ be $n+1$ numbers. There 
exists an $n$-simplex $\mathscr{A}$ such that $v_{n-1}\left(\mathscr{A}_{i}\right)=f_{i}, i \in \mathscr{J}$, if and only if $f_{0}<\Sigma^{\prime} f_{i}$.

Proof. If $\mathscr{A}$ is a simplex with $v_{n-1}\left(\mathscr{A}_{i}\right)=f_{i}, i \in \mathscr{J}$, and $f_{i}$ is a vector normal to $\mathscr{A}_{i}$ in the outbound direction with magnitude $f_{\text {t }}, i \in \mathscr{F}$, then [8]

$$
-\boldsymbol{f}_{0}=\sum^{\prime} \boldsymbol{f}_{\imath}
$$

(Applying the Gauss divergence theorem to a constant function on $\mathscr{A}$ and its boundary gives this result immediately.) The $f_{i}$ are called the vector hyperareas of $\mathscr{A}$. Since the $f_{i}, i \in \mathscr{I}^{\prime}$, are linearly independent, the triangle inequality for vectors yields $f_{0}<\Sigma^{\prime} f_{i}$.

Conversely, if $f_{0}<\Sigma^{\prime} f_{i}$, we can construct a skew $(n+1)$-gon $P_{0} P_{1} \cdots P_{n} P_{n+1}$ where $P_{n+1}=P_{0}$ with $P_{i} P_{i+1}=f_{i}, i \in \mathscr{I}$. For an arbitrary point $Q$, define $Q_{i}$ by $\overrightarrow{Q Q}_{i}=\vec{P}_{i}$ and construct a simplex $\mathscr{B}$ by taking a prime perpendicular to line $Q Q_{i}$ at $Q_{i}, i \in \mathscr{I}$. For some $k_{i}, i \in \mathscr{I}$, the vectors $k_{i} \overrightarrow{Q Q}$, are normal to the faces $\mathscr{B}_{i}$ of $\mathscr{B}$ in the outbound direction with magnitude equal to $v_{n-1}\left(B_{i}\right)$. In view of (5.1) we have

$$
-k_{0} \overrightarrow{Q Q}_{0}=\sum^{\prime} k_{i} \overrightarrow{Q Q}_{i}
$$

However, $\Sigma \overrightarrow{Q Q}_{1}=\sum{\overrightarrow{P_{i} P_{i+1}}}=\mathbf{0}$ so

$$
-\overrightarrow{Q Q}_{0}=\sum \overrightarrow{Q Q}_{1}
$$

Since, by skewness, the vectors $\overrightarrow{Q Q}_{1}, i \in \mathscr{I}^{\prime}$, are independent, comparison of (5.2) and (5.3) shows that all the $k_{i}$ must be equal. Thus the $v_{n-1}\left(\mathscr{B}_{l}\right)$ are proportional to the $Q Q_{l}$, i.e. to the given $f_{i}$. A dilatation completes the construction and the proof.

It is clear from the construction that if the $f_{i}$ are equal there is still a great deal of freedom and the resulting simplex need not be regular.

Corollary 5.2. Let $f_{i}, i \in \mathscr{I}$, be given vectors. There exists an $n$-simplex $\mathscr{A}$ whose vector hyperareas are the $f_{i}$ if and only if $\Sigma f_{i}=\mathbf{0}$.

Corollary 5.3 [2]. For any n-simplex $\mathscr{A}$, with $v_{n-1}\left(\mathscr{A}_{1}\right)=f_{1}, i \in \mathscr{I}$, $f_{0}^{2}=\Sigma^{\prime} f_{i}^{2}+2 \Sigma_{1 \leqq i<j \leqq n} f_{i} f_{j} \cos \left(a_{i}, a_{1}\right)$. 
Proof. Take the dot product of each side of (5.1) with itself.

Corollary 5.4. Theorem of Pythagoras. If $\mathscr{A}$ is a right simplex with orthocenter at $A_{0}$, then $f_{0}^{2}=\Sigma^{\prime} f_{i}^{2}$.

Since the altitudes are inversely proportional to the $(n-1)$ volumes of the faces, we have the following.

COROLLARY 5.5. Let $0 \leqq h_{0} \leqq h_{1} \leqq \cdots \leqq h_{n}$ be numbers. There exists an n-simplex $\mathscr{A}$ such that the altitude from $A_{i}$ has length $h_{i}, i \in \mathscr{I}$, if and only if $h_{0}^{-1}<\Sigma^{\prime} h_{i}^{-1}$.

Corollary 5.6. If $\mathscr{A}$ is a right simplex with orthocenter $H=A_{0}$, then $h_{0}^{-2}=\Sigma^{\prime} h_{i}^{-2}=\Sigma^{\prime} A_{0} A_{i}^{-2}$.

In conclusion we remark that Corollary 5.2 has the following physical interpretation. If a joint in a three dimensional structure is to be in equilibrium under the action of four forces, it is possible to construct a tetrahedral bearing plate with faces normal to the forces and such that the bearing load is uniform.

6. Solution to problem (iv). Essentially we are seeking the parameters of an orthocentric simplex given its altitudes.

Lemma 6.1. Let $\mathscr{A}$ be an orthocentric simplex with parameters, altitudes and hyperareas $a_{i}, h_{i}, f_{i}, i \in \mathscr{I}$. Then the following conditions are equivalent.

$$
\begin{aligned}
& a_{0} \leqq a_{1} \leqq \cdots \leqq a_{n}, \\
& h_{0} \leqq h_{1} \leqq \cdots \leqq h_{n}, \\
& f_{0} \geqq f_{1} \geqq \cdots \geqq f_{n}
\end{aligned}
$$

and strict inequality corresponds to strict inequality.

Proof. Assume (6.1). Since the $f_{i}$ are inversely proportional to the $h_{i}$, we need only show the equivalence of (6.1) and (6.2). If $a=0$, then $\mathscr{A}$ is a right simplex, $a_{0}=0, h_{i}^{2}=A_{0} A_{i}^{2}=a_{i}, i \in \mathscr{I}^{\prime}$, and $h_{0}^{-2}=\Sigma^{\prime} h_{i}^{-2}$ so the equivalence is immediate. If $a \neq 0$, then by (1.7) and (1.4)

$$
\begin{aligned}
h_{i}^{2} & =\left(A_{i} H+H H_{i}\right)^{2}=A_{i} H^{2}+2 A_{i} H \cdot H H_{i}+H H_{i}^{2} \\
& =A_{i} H^{2}+2 a+a^{2} / A_{i} H^{2}=\left(a_{i}-a\right)+2 a+a^{2} /\left(a_{i}-a\right) \\
& =a_{i}^{2} /\left(a_{i}-a\right), \quad i \in \mathscr{I} .
\end{aligned}
$$


That is, $a h_{i}^{-2}=a a_{i}^{-1}\left(1-a a_{i}^{-1}\right), i \in \mathscr{I}$, subject to $\sum a a_{i}^{-1}=1$. With $y_{i}=$ $a h_{i}^{-2}$ and $x_{i}=a a_{1}^{-1}, i \in \mathscr{I}$, we have

$$
y_{i}=x_{i}\left(1-x_{i}\right) \text { subject to } \Sigma x_{i}=1 .
$$

Suppose $a>0$ so $x_{i}>0, i \in \mathscr{I}$. If $x_{i} \leqq \frac{1}{2}, i \in \mathscr{I}$, then clearly sign $\left(y_{i}-y_{j}\right)=\operatorname{sign}\left(x_{i}-x_{j}\right)$. If $x_{0}>\frac{1}{2}$, then $x_{0}+x_{i}<1$ implies that $x_{0}-\frac{1}{2}<$ $\frac{1}{2}-x_{i}, i \in \mathscr{I}^{\prime}$. That is, $x_{0}$ is closest to $\frac{1}{2}$ and $y_{0}$ is the largest. For $i \in \mathscr{I}^{\prime}$, $x_{i}<\frac{1}{2}$ and the properties follow.

Suppose $a<0$ so $x_{i}<0, i \in \mathscr{I}^{\prime}$, and $x_{0}>1$. Since $x_{0}>\frac{1}{2}$, the order properties of the $x_{i}$ and $y_{i}$ again follow and the equivalence of (6.1) and (6.2) is immediate.

COROLlary 6.2. An orthocentric simplex with equal altitudes or hyperareas is regular.

Corollary 6.3. In an orthocentric simplex $\mathscr{A}$, the orthocenter $H$ lies closer to a face than to the corresponding vertex on all except possibly the shortest altitude.

Proof. If $A_{0}$ is interior to $H A_{1} \cdots A_{n}$, then for $i \in \mathscr{I}^{\prime}, H$ and $A_{i}$ are on opposite sides of $\mathscr{A}_{i}$. If $\mathscr{A}$ is a right simplex, the result is trivial. If $H$ is interior to $\mathscr{A}$, then from (1.3) it follows that that barycentric coordinates of $H$ with respect to $\mathscr{A}$ are $\left(a a_{0}^{-1}, \cdots, a a^{-1}\right)$ and only the largest of these can exceed $\frac{1}{2}$.

We now state the solution to problem (iv).

THEOREM 6.4. Let $0<h_{0} \leqq h_{1} \leqq \cdots \leqq h_{n}$ be given numbers with $h_{0}^{-1}<\Sigma^{\prime} h_{i}^{-1}$. Then there is an orthocentric simplex $\mathscr{A}$ whose $i$ th altitude $A_{i} H_{i}$ has length $h_{i}, i \in \mathscr{I}$. A is unique to within an isometry.

Further, let

$$
f(a)=\epsilon\left(1-4 h_{0}^{-2} a\right)^{\frac{1}{2}}+\sum^{\prime}\left(1-4 h_{i}^{-2} a\right)^{\frac{1}{2}}-(n-1) .
$$

If $h_{0}^{-2}<\Sigma^{\prime} h_{i}^{-2}$, then the orthocenter $H$ will be interior to $\mathscr{A}$ and the parameter $a$ is the unique positive root of $f(a)=0$ where $\epsilon= \pm 1$ according as $\Sigma^{\prime}\left(1-h_{0}^{2} h_{i}^{-2}\right)^{\frac{1}{2}}-(n-1)$ is negative or positive. If $h_{0}^{-2}>$ $\Sigma^{\prime} h_{i}^{-2}$, then $A_{0}$ will be interior to $H A_{1} \cdots A_{n}$ and $a$ is the unique negative root of $f(a)=0$ with $\epsilon=-1$. In either case,

$$
a_{0}=\frac{1}{2} h_{0}^{2}\left[1+\epsilon\left(1-4 a h_{0}^{-2}\right)^{\frac{1}{1}}\right]
$$

and 


$$
a_{i}=\frac{1}{2} h_{i}^{2}\left[1+\left(1-4 a h_{i}^{-2}\right)^{\frac{1}{2}}\right], \quad i \in \mathscr{I}^{\prime} .
$$

If $h_{0}^{-2}=\Sigma^{\prime} h_{i}^{-2}$, then $\mathscr{A}$ is a right simplex with $H=A_{0}, a=a_{0}=0$ and $a_{i}=h_{i}^{2}, i \in \mathscr{I}^{\prime}$.

Proof. First we consider a right simplex with orthocenter $H=$ $A_{0}$. From facts (1.3) and (1.2) we see that $a=a_{0}=0$ and for $i \in \mathscr{I}^{\prime}$, the altitude $A_{i} H_{t}$ coincides with the edges $A_{0} A_{i}$ so $a_{i}=A_{0} A_{i}^{2}-a_{0}=$ $h_{i}^{2}$. From Corollary 5.6 we have $h_{0}^{-2}=\Sigma^{\prime} h_{i}^{-2}$.

Suppose now $\mathscr{A}$ is an orthocentric simplex which is not right. Solving (6.4) for $a_{i}$ we find

$$
a_{i}=\frac{1}{2} h_{i}^{2}\left[1+\epsilon_{i}\left(1-4 a h_{i}^{-2}\right)^{\frac{1}{2}}\right], \quad \epsilon_{i}= \pm 1
$$

and

$$
1-2 a a_{i}^{-1}=\epsilon_{i}\left(1-4 a h_{i}^{-2}\right)^{\frac{1}{2}}
$$

It follows from Lemma 6.1 that $a_{0} \leqq a_{1} \leqq \cdots \leqq a_{n}$ and that $a a_{i}^{-1}<\frac{1}{2}$ for $i \in \mathscr{I}^{\prime}$, so $\epsilon_{i}=+1, i \in \mathscr{I}^{\prime}$. Summing (6.6) over $i$, using $\sum a a_{i}^{-1}=1$, and replacing $\epsilon_{0}$ by $\epsilon$ we obtain $f(a)=0$ where $f(a)$ is given by (6.5). We assert that if $h_{0}^{-2}<\Sigma^{\prime} h_{i}^{-2}$, then only one choice of $\epsilon$ will yield a positive root and for that choice the solution is unique; and if $h_{0}^{-2}>\Sigma^{\prime} h_{i}^{-2}$, then neither choice of $\epsilon$ will yield a positive root.

Suppose $\epsilon=-1$. Rearranging,

$$
\begin{aligned}
f(a) & =-\left[\left(1-4 h_{0}^{-2} a\right)^{\frac{1}{2}}-1\right]+\sum^{\prime}\left[\left(1-4 h_{i}^{-2} a\right)^{\frac{1}{2}}-1\right] \\
& =4 a\left\{\frac{h_{0}^{-2}}{\left(1-4 h_{0}^{-2} a\right)^{\frac{1}{2}}+1}-\sum^{\prime} \frac{h_{i}^{-2}}{\left(1-4 h_{i}^{-2} a\right)^{\frac{1}{2}}+1}\right\}
\end{aligned}
$$

and $f(0)=0$. Further, the derivative

$$
f^{\prime}(a)=2\left\{\frac{h_{0}^{-2}}{\left(1-4 h_{0}^{-2} a\right)^{\frac{1}{2}}}-\sum^{\prime} \frac{h_{i}^{-2}}{\left(1-4 h_{i}^{-2} a\right)^{\frac{1}{2}}}\right\}
$$

and $f^{\prime}(0)=2\left(h_{0}^{-2}-\Sigma^{\prime} h_{i}^{-2}\right)$. For $i \in \mathscr{I}^{\prime}, h_{0}^{-2} \geqq h_{i}^{-2}$ so for $a>0$

$$
\frac{\left(1-4 h_{0}^{-2} a\right)^{\frac{1}{2}}}{\left(1-4 h_{i}^{-2} a\right)^{\frac{1}{2}}} \leqq \frac{\left(1-4 h_{0}^{-2} a\right)^{\frac{1}{2}}+1}{\left(1-4 h_{i}^{-2} a\right)^{\frac{1}{2}}+1}
$$

A term by term comparison shows that

$$
2 a f^{\prime}(a)\left(1-4 h_{0}^{-2} a\right)^{\frac{1}{2}} \geqq f(a)\left[\left(1-4 h_{0}^{-2} a\right)^{\frac{1}{2}}+1\right] .
$$


Thus $f^{\prime}(a)>0$ whenever $f(a) \geqq 0$ (unless all the $h_{i}$ are equal in which case it is clear the only root is zero) so there is at most one positive root. If $h_{0}^{-2}>\Sigma^{\prime} h_{i}^{-2}$, then $f^{\prime}(0)>0$ and $f(a)$ and $f^{\prime}(a)$ are positive $a>0$, so there are no positive roots. Suppose $h_{0}^{-2}<\Sigma^{\prime} h_{i}^{-2}$ so $f^{\prime}(a)<$ 0 . Thus if the right endpoint value $f\left(\frac{1}{4} h_{0}^{2}\right) \geqq 0$ there is precisely one positive root, and if $f\left(\frac{1}{4} h_{0}^{2}\right)<0$ there is no positive root.

Suppose $\epsilon=1$. Then $f(0)=2$ and $f(a)$ is monotone decreasing so if $f\left(\frac{1}{4} h_{0}^{2}\right)>0$ there is no positive root, and if $f\left(\frac{1}{4} h_{0}^{2}\right) \leqq 0$ there is a unique positive root. Further, if $h_{0}^{-2}>\Sigma^{\prime} h_{i}^{-2}$, then $h_{0}<h_{i}, i \in \mathscr{I}^{\prime}$, and

$$
f\left(\frac{1}{4} h_{0}^{2}\right)=1-h_{0}^{2} \sum^{\prime} \frac{h_{i}^{-2}}{1+\left(1-h_{0}^{2} h_{i}^{2}\right)^{\frac{1}{2}}}>1-h_{0}^{2} \sum^{\prime} h_{i}^{-2} \geqq 0
$$

so there are no positive roots here either.

The case $\epsilon=1$ corresponds to $a a_{0}^{-1}<\frac{1}{2}$, i.e. $H$ nearer to $\mathscr{A}_{0}$ and the case $\epsilon=-1$ corresponds to $H$ nearer $A_{0}$. If $\frac{1}{4} h_{0}^{2}$ is a root, $H$ is the midpoint of $A_{0} H_{0}$, and the sign of $\epsilon$ is immaterial, a petty infringement on uniqueness.

The search for negative roots is considerably simplified by the fact that $a<a_{0}<0$ and $a_{i}>0, i \in \mathscr{I}^{\prime}$, together with (6.6) imply $\epsilon_{0}=-1$ and $\epsilon_{i}=+1, i \in \mathscr{I}^{\prime}$. In this case we observe that at $-\infty$,

$$
f(a) \sim 2(-a)^{\frac{1}{2}}\left[\sum^{\prime} h_{i}^{-1}-h_{0}^{-1}\right]>0
$$

and it follows as above that there will be a negative root if and only if

$$
f^{\prime}(0)=2\left(h_{0}^{-2}-\sum^{\prime} h_{i}^{-2}\right) \geqq 0 .
$$

\section{Solution to the remaining problems.}

THEOREM 7.1. Let $f_{i}, i \in \mathscr{I}$, be numbers. The simplex $\mathscr{A}$ of maximum volume for which $v_{n-1}\left(a_{i}\right)=f_{i}, i \in \mathscr{I}$, is orthocentric with altitudes proportional to $f_{i}^{-1}, i \in \mathscr{I}$. If $Q$ is a given point, the simplex $\mathscr{B}$ of maximal volume such that $Q B_{i}=f_{i}, i \in \mathscr{I}$, and that $Q$ is the centroid of $\mathscr{B}$ is constructed by setting the vectors $\overrightarrow{Q B}_{i}$ equal to the vector hyperareas of $\mathscr{A}$.

THEOREM 7.2. Let $s>0$ and $p \geqq p_{n}$ be fixed, where $p_{n} \leqq 1$ is given by (4.4). The n-simplex $\mathscr{C}$ of maximal volume such that

$$
\frac{1}{n+1} \sum C_{i} C_{1+1}^{p}=s^{p}
$$

is a regular simplex. 
Proofs. If the given hyperareas satisfy the extended triangle inequality there are simplexes whose faces have the prescribed volume and it is clear that a maximizing simplex exists. (The isoperimetric inequality states that the $n$-volume of a simplex with these hyperareas is less than that of a sphere with surface $\Sigma f_{i}$, but the following crude estimate will also suffice: the incribed sphere of any such simplex obviously has surface less than $\Sigma f_{i}$ and the $n$-volume of the simplex is $1 / n \sum f_{i}$ times its radius.) Let $\mathscr{A}$ be any simplex with $v_{n-1}\left(\mathscr{A}_{i}\right)=f_{i}$, $i \in \mathscr{I}$. Let $h_{i}=A_{i} H_{i}, i \in \mathscr{I}$, and let $v=v_{n}(\mathscr{A})$. Let $Q$ be any point and construct $Q B_{i}$ perpendicular to $\mathscr{A}_{i}$ with $Q B_{i}=f_{i}, i \in \mathscr{I}$. Since $\Sigma \overrightarrow{Q B}_{i}=0$ it follows that $Q$ is the centroid of the simplex $\mathscr{B}=$ $B_{0} B_{1} \cdots B_{n}$. Let $\mathscr{B}_{0}$ be the simplex $Q B_{1} \cdots B_{n}$. Then (cf. [5, p. 4])

$$
(n ! v)\left(n ! v_{n}\left(\mathscr{B}_{0}\right)\right)=\operatorname{det}\left(\overrightarrow{A_{0} A_{i}} \cdot \overrightarrow{Q B_{j}}\right)=\prod^{\prime} \overrightarrow{A_{0} A_{i}} \cdot \overrightarrow{Q B_{i}}=n^{n} v^{n}
$$

since the nondiagonal elements vanish and $\overrightarrow{A_{0} A_{i}} \cdot \overrightarrow{Q B_{i}}=h_{i} Q B_{i}=h_{i} f_{i}=$ $n v$. Thus

$$
v_{n}(\mathscr{B})=(n+1) v_{n}\left(\mathscr{B}_{0}\right)=(n !)^{-2}(n+1) n^{n} v^{n-1} .
$$

Now construct $\mathscr{C}=C_{0} C_{1} \cdots C_{n}$ with $C_{0}$ arbitrary and $\overrightarrow{C_{i-1}} C_{i}=\overrightarrow{Q B}_{i}$, $i \in \mathscr{I}^{\prime}$. Thus

$$
\sum_{i=1}^{k} \overrightarrow{Q B_{i}}=\sum_{i=1}^{k} \overrightarrow{C_{i-1} C_{i}}=\overrightarrow{C_{0} C_{k}}, \quad k \in \mathscr{I}^{\prime},
$$

and in particular $\overrightarrow{C_{n} C_{0}}=-\Sigma^{\prime} \overrightarrow{Q B_{i}}=\overrightarrow{Q B_{0}}$. If the vectors $\overrightarrow{C_{0} C_{k}}, k \in \mathscr{I}^{\prime}$, are chosen as a basis, the volume of $\mathscr{B}_{0}$ relative to the volume of $\mathscr{C}$ is a lower triangular determinant with l's on and below the main diagonal. It follows that $v_{n}(\mathscr{C})=v_{n}\left(\mathscr{B}_{0}\right)$ so if we maximize $v_{n}(\mathscr{C})$, we maximize $v_{n}(\mathscr{B})$ and hence $v_{n}(\mathscr{A})$. Allowing $C_{0}$ to vary on the intersection of the spheres determined by $C_{n} C_{0}=Q B_{0}=f_{0}$ and $C_{0} C_{1}=Q B_{1}=f_{1}$, it is clear that the altitude from $C_{0}$ to the opposite face $\mathscr{C}_{0}=C_{1} C_{2} \cdots C_{n}$, and hence $v_{n}(\mathscr{C})$, is maximized when the plane $C_{n} C_{0} C_{1}$ is perpendicular to $\mathscr{C}_{0}$, i.e. $Q B_{0} B_{1}$ is perpendicular to $Q B_{2} \cdots B_{n}$ so $A_{2} \cdots A_{n}$ is perpendicular to the lines $A_{0} A_{1}$. Since the labelling is arbitrary, each edge of the maximal $\mathscr{A}$ is perpendicular to the opposite face, so $\mathscr{A}$ is orthocentric (fact 1.1). The orthocentric simplex with altitudes $f_{i}^{-1}, i \in \mathscr{I}$, constructed as in the solution to (iv), is similar to $\mathscr{A}$ and $\mathscr{A}$ is obtained by a dilatation. This completes the solution to (v). $\mathscr{B}$ can be constructed immediately, which gives the solution to (vi). 
Turning to problem (vii), we suppose we want to maximize $v_{n}(\mathscr{C})$ given the sum of the $p$ th powers, $p \geqq p_{n}$ as in the solution of (iii), of the edges of the skew polygon $C_{0} C_{1} \cdots C_{n} C_{0}$. This is equivalent to maximizing $v_{n}(\mathscr{B})$ given the sum $\Sigma Q B_{i}^{p}$ with the constraint that $Q$ is the centroid of $\mathscr{B}$. But we have already shown in solving (iii) that the unconstrained maximum is attained when $\mathscr{B}$ is a regular simplex and $Q$ is its center. So $\mathscr{C}$ is also regular; this completes the solution of (vii). When $p=1$, the sum of the $p$ th powers of the edges of $\mathscr{C}$ is just the perimeter of the polygon, so we have the result of [13].

\section{REFERENCES}

1. M. M. Ali, On some extreme simplexes, Pacific J. Math., 33 (1970), 1-14.

2. P. Bartos, Cosine theorems on simplexes in $E^{n}$ (Czech), Casopis Pest. Mat., 95 (1970), 150-154.

3. J. Berkes, Einfacher Beweis und Verallgemeinerung einer Dreiecksgleichung, Elem. Math., 22 (1967), 135-136.

4. C. W. Borchardt, Uber die Aufgabe des Maximum, welche der Bestimmung des Tetraeders von grossten Volumen bei gegebenen Flächeninhalt der Seitenflächen fur mehr als drei Dimensionen entspricht, Math. Abh. Akad. Wis. Berlin, 1866, pp. 121-155 $\equiv$ Gesammelte Werke, Berlin, 1888, pp. 201-232.

5. L. Brand, Vector Analysis, Wiley, New York, 1957.

6. M. M. Day, Polygons circumscribed about closed convex curves, Trans. Amer. Math. Soc., 62 (1947), 315-319.

7. E. Egervary, On orthocentric simplexes, Acta Lith. Sci. Szeged, 9 (1940), 218-266.

8. L. Gerber, The altitudes of a simplex are associated, Math. Mag., 46 (1973), 155-157.

9. - Associated and perspective simplexes, Trans. Amer. Math. Soc., 201 (1975), 43-55.

10. G. H. Hardy, J. E. Littlewood, G. Polya, Inequalities, Cambridge U. Press, London, 1934.

11. J. L. Lagrange, Solutions analytiques de quelques problèmes sur les pyramides triangulaires, Nouv. Mém. Acad. Sci. Berlin, 1773, pp. 149-176 $\equiv$ Ouvres completes III, pp. 659-692.

12. M. A. Marmion, Solution to Question 3253, Mathesis, 62 (1953), 69-72.

13. B. Misek, The simplex polygon with the greatest volume of its convex hull (Czech), Casopis Pest. Mat., 84 (1959), 99-104.

14. C. M. Petty and D. Waterman, An extreme theorem for $n$-simplexes, Monatsh. Math., 59 (1955), 320-322.

15. D. Slepian, The content of some extreme simplexes, Pacific J. Math., 31 (1969), 795-808.

Received August 2, 1973.

ST. JOHN'S UNIVERSITY 
